\title{
Experimental Study: Link Quality and Deployment Issues in Wireless Sensor Networks
}

\author{
Monique Becker, Andre-Luc Beylot, Riadh Dhaou, \\ Ashish Gupta, Rahim Kacimi, and Michel Marot* \\ Institut TELECOM, CNRS-SAMOVAR, 9 Rue Charles Fourier, Evry, France \\ \{Monique.Becker, Ashish.Gupta, Michel. Marot\}@it-sudparis .eu \\ University of Toulouse, IRIT-ENSEEIHT, 2 Rue Charles Camichel, Toulouse, France \\ \{Andre-Luc.Beylot, Riadh.Dhaou, Rahim.Kacimi\}@enseeiht.fr
}

\begin{abstract}
In this paper, we highlight the extent of the effects of topological specificities on the deployed solutions, which can be useful to refine already proposed models as well as to carry out protocol tuning or adjustments. We present, an intensive experimental study on wireless Link Quality Indicator (LQI). Using Moteiv's Tmote Sky sensors, we deployed multiHopLQI algorithm of TinyOS in various network configurations: homogeneous and heterogeneous; straight-line and grid topologies with various transmission power levels and distances.

Initially, we study LQI time-varying and try to understand the relationship between transmission power level, distance and link quality and present how some random disturbances due to external (physical changes) or internal phenomena (node movement,power variation) may affect the dynamics of the network. Later, we address impacts and side effects of position and power transmission level of some important nodes in the network like the Base Station in such LQI based algorithms.
\end{abstract}

Keywords: Wireless Sensor Networks, CC2420 Radio, Link Quality Indicator, Transmission power.

\section{Introduction}

Most of the sensor applications are designed to use simple, cheap, tiny devices with limited battery power. Furthermore, when real sensors are deployed, usually they do not have access to GPS (which is high energy consuming as well as expensive). Therefore, they flood the network with messages like ROUTE_REQUEST and then wait for the replies from the neighboring sensors to identify their exact location in the network. Based on the replies, they also construct their neighbor table or routing table to build the network as well as topology.

The sensors do not know the exact physical locations of the other sensor nodes, the decision which sensor is near or far is dependent upon the received signal quality or in case of our experiments Link Quality Indicator (LQI). For

\footnotetext{
* The authors name appear in alphabetical order and the experimental work has been jointly conducted by Rahim Kacimi and Ashish Gupta.
} 
each received packet, this value is obtained through Chipcon CC2420 [1] radio module provided in the Moteiv's Tmote Sky sensor [2]. As per the matrix of Chipcon, the higher the LQI value is, the better the link quality between the two nodes is. Therefore, in these sensors, if the LQI between two sensors is above a given threshold, they can communicate directly, taking into account the overall network topology. In this paper, we consider scenarios (presented in the following section) where the sensors calculate the LQI between them and the top 3 neighbors and construct the neighbor table accordingly, with number of hops (distance) from the Base Station (BS). The choice of the next hop is based on the link cost estimation, in order to connect with the BS. The link cost depends jointly on the LQI and on the minimum number of hops algorithm. The most interesting aspect in any sensor network is the transmission power of the sensor, a major component of energy consumption in any sensor. Higher transmission power leads to better signal quality over a large area, nonetheless resulting in higher energy consumption and vice-versa.

The remainder of the paper is organized as follows. Section 2 introduces prior works and problems. Then, we discuss our experimental methodology in Section 3. In Section 4, we present and analyze the experimental results. And finally, Section 5 concludes the paper.

\section{Problem and Background}

Last few years have witnessed the tremendous leap in sensor network domain. Indeed, researchers try to exploit all the parameters that this domain provides to improve the performance criteria of the proposed solutions, protocols, and algorithms. In [3], authors present a resource-aware and link quality based routing metric for wireless sensor and actor networks in order to adapt to variable wireless channel conditions in such heterogeneous networks. In the field of localization, Blumenthal et al. use the LQI to estimate a distance from a node to some reference points [4. More currently, the experimental/deployment analysis become one of the forefront subject in WSN field. Recent experimental studies [5], [6], 7], 8] and [9] have shown that in real sensor network deployments, wireless link quality varies over space and time. In [6], authors investigated performance issues related to node placement, packet rate and distance. In [5], Wahba et al. used two motes and evaluated link quality over distance and various power levels. Polastre et al. [10] presented preliminary evaluation results for Telos motes (based on CC2420) and suggested that the average LQI was a better indicator of packet reception rate $(\mathrm{PRR})$. In all the work, authors have taken into account the homogeneous nature of the network, where all the nodes have equal transmission power. Higher energy emission leads to better signal over a large area, resulting in higher energy consumption or vice-versa.

The most interesting aspect in any sensor network is the transmission power of the sensor, a major component of energy consumption in any sensor. This paper compares the various homogeneous and heterogeneous scenarios (described in next section) and their effect on Link Quality and hence on the connectivity of the network. 
Thus, a sufficient reason for our interest in the link quality is to answer the following questions. Is this parameter time-varying? What are the factors of this variation? How LQI depends on transmission power and distances between the nodes? And finally, what is its impacts on routing and network topology?

\section{$3 \quad$ Experimental Set-Up}

In order to experience and understand how few fundamental aspects of deployment can influence the sensor network as a whole, let us analyze some real time deployment issues. We have conducted 40 different scenarios and have recorded observations for more than 800 minutes (grand total of all scenarios) per sensor. All these scenarios are different either in terms of number of nodes, distance between the nodes, transmission power level of nodes, transmission power level of Base Station (BS) or finally, in terms of topology i.e. straight-line/grid (Fig. 1). All the scenarios are conducted in indoor conditions. The experiments are performed at several power levels.

In fact, all these scenarios helped us to compare several as well as relevant configurations for a given sensor network. We started with simple straight line topology, observed the network with time, node displacement, positioning, connectivity, etc. Then applied those observations by adding node redundancy (gridtopology) to the network.

Tmote Sky is a small platform including a microcontroller operating at $8 \mathrm{MHz}$, $48 \mathrm{~K}$ of ROM, $10 \mathrm{~K}$ of RAM, a $2.4 \mathrm{GHz}$ ZigBee wireless transceiver, and a USB interface for device programming and logging. Each device operates on $2 \mathrm{AA}$ batteries. Tmote Sky node provides an interface to parameterize its transmission power. The parameter varies from 1 (-25 dBm, minimum Transmission Power Level (TPL)) to 31 (0 dBm, the maximum TPL). Therefore, just by varying the TPL parameter transmission power can be increased or decreased. Additionally, in all the scenarios only printed antenna on the sensor has been used (no additional external antenna). Furthermore, all the sensors are placed on the floor.

All scenarios, as described in Table 1 later are based on following assumptions:

- Sensors usually have low quality of radio antenna.

- Deployment in an area with steady environment is not possible.

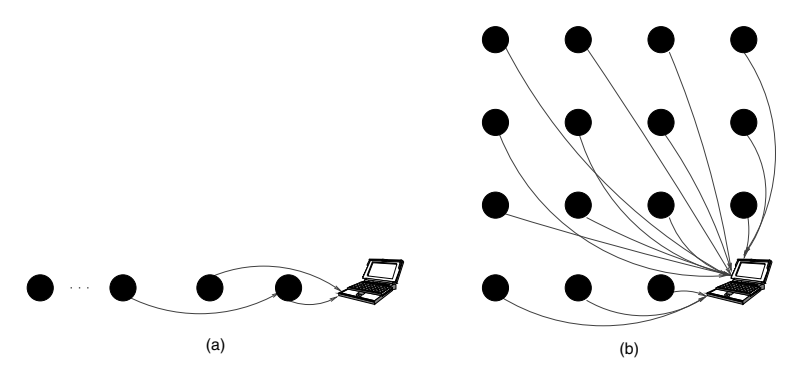

Fig. 1. Straight-line (a) and Grid (b) deployment 
Table 1. Scenario Description

\begin{tabular}{|c|c|c|c|c|}
\hline Scenario & Nodes count & BS-TPL & Node-TPL & Distance \\
\hline 1 & 12 & 31 & 25 & 3 \\
\hline 2 & 7 & 31 & 25 & 6 \\
\hline 3 & 7 & 25 & 25 & 6 \\
\hline 4 & 5 & 25 & 25 & 9 \\
\hline 5 & 5 & 31 & 25 & 9 \\
\hline 6 & 12 & 31 & 20 & 3 \\
\hline 7 & 12 & 20 & 20 & 3 \\
\hline 8 & 7 & 31 & 20 & 6 \\
\hline 9 & 7 & 20 & 20 & 6 \\
\hline 10 & 5 & 20 & 20 & 9 \\
\hline 11 & 5 & 31 & 20 & 9 \\
\hline 12 & 12 & 31 & 15 & 3 \\
\hline 13 & 12 & 15 & 15 & 3 \\
\hline 14 & 7 & 15 & 15 & 6 \\
\hline 15 & 5 & 15 & 15 & 9 \\
\hline 16 & 5 & 31 & 15 & 9 \\
\hline 17 & 7 & 31 & 15 & 6 \\
\hline 18 & 12 & 31 & 10 & 3 \\
\hline 19 & 12 & 10 & 10 & 3 \\
\hline 20 & 7 & 31 & 10 & 6 \\
\hline 21 & 7 & 10 & 10 & 6 \\
\hline 22 & 5 & 31 & 10 & 9 \\
\hline 23 & 5 & 10 & 10 & 9 \\
\hline 24 & 12 & 31 & 5 & 3 \\
\hline 25 & 12 & 5 & 5 & 3 \\
\hline 26 & 7 & 31 & 5 & 6 \\
\hline 27 & 7 & 5 & 5 & 6 \\
\hline 28 & 5 & 31 & 5 & 9 \\
\hline 29 & 5 & 5 & 5 & 9 \\
\hline
\end{tabular}

\section{Analysis and Observation}

In Scenarios 1 to 29 (Tab. 1), nodes are placed in 2m (approx.) wide indoor corridor (in straight line, direct visibility) along the wall. Further, the area is open to public and have experienced frequent movements of people during the measurements.

In these scenarios, we varied the number of nodes (respectively, 12, 7 then 5 , including BS), separated by 3, 6, and 9 meters respectively. For each set of above parameters, we have used two different sets of Transmission Power Level (TPL), namely SetMax $\{31\}$ and SetLow $\{25,20,15,10,5\}$.

\subsection{Real Time Evolution}

The channel quality of a given sensor network is dynamic i.e., not only it is being affected by the limited battery of sensors but also by the periodic/random 


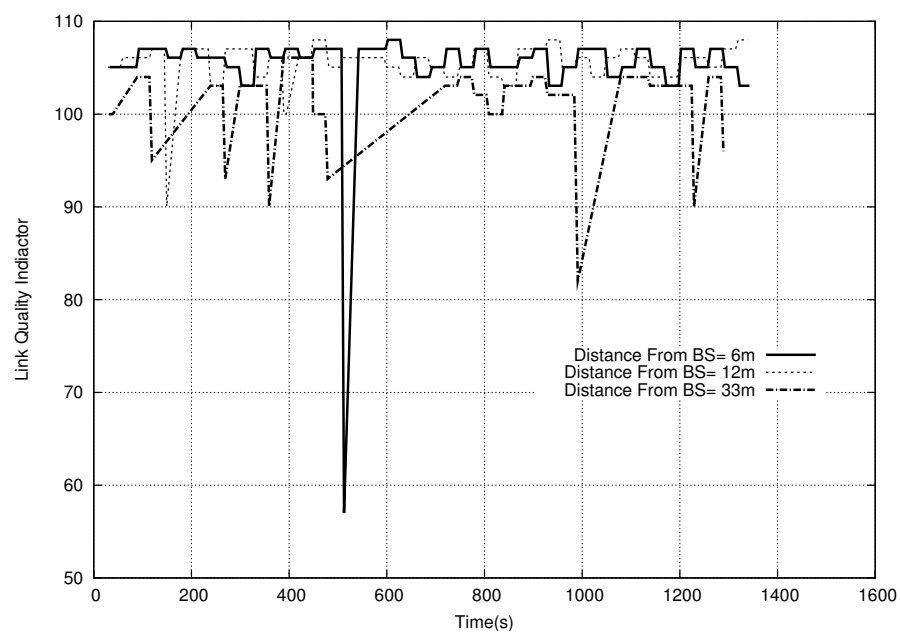

Fig. 2. Real time evolution of LQI

change in the physical properties of the channel, e.g. a group of people passing around the sensors can easily change the dynamics of the network. In Fig. 2, we plan to summarize this effect and will discuss the Scenario 1. Whenever, there has been a movement of group of people, in and around the network, we have experienced connectivity problems. The troughs which are being presented in Fig. 2, represent the deterioration of communication channel. Furthermore, the sharper curves leads to change in the connectivity and topology in the network. Let us remember, only the LQI readings between the sensor nodes and the BS are being discussed. In fact, it shows network instability and its vulnerability to physical medium, even as in Scenario 1, considering connectivity range, nodes are very powerful and more are or less are very near.

\subsection{Impact of the Position of the Base Station}

In most of the sensor networks, the role of a BS is to collect data and send it to a remote server or end-user. The BS can be selected statically or dynamically. The LQI usually determines the connectivity between the various nodes. Here, we will discuss Scenario 24. In this scenario, we have 12 nodes including BS. Each node is separated by 3 meters and all the nodes are in straight line (direct visibility). Fig. 3 presents the LQI values between various sensors and the BS. We have observed that troughs deal with the discontinuity in the network and shaper troughs in LQI reading lead to disruption of communication channel/link. Furthermore, positioning of the BS can have a subtle effect on the performance of BS. Also, all the nodes are placed on the floor next to wall. We have run this scenario for over 1200 seconds. Even though, there are another 6 nodes (excluding BS) in the network. For Clarity reasons, we present the relevant results only for few nodes. Initially, we have observed that, the node which is 33 meters away from the BS, is not connected directly with BS. Thereafter, (from time 50-500 


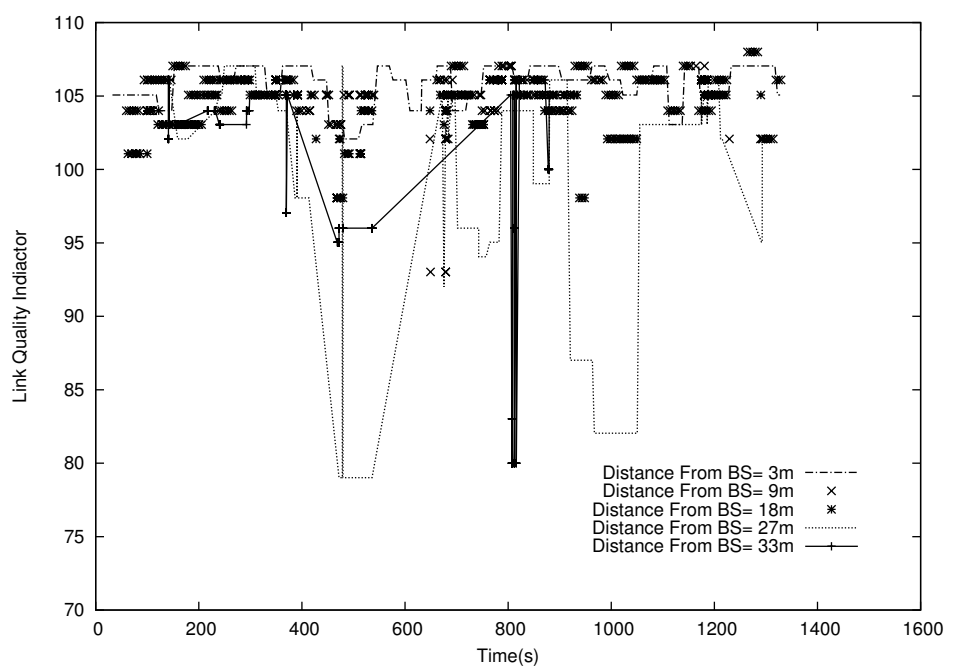

Fig. 3. Impact of position of Base Station

seconds) we raise the position of the BS by about 0.5 meter from the floor. Again, we can see from Fig. 3, merely, by raising the position of BS with respect to other sensors, we observe the major shift in LQI values. Later on, we play with BS with intermittingly raising and lowering the position of BS and finally, at around time $=800$ seconds we end this procedure. Between these periods, we can easily distinguish the various LQI troughs being made repeatedly. And once, we are over with this process, farthest node is connected via multiple hops with BS i.e. no more direct connectivity with BS. The fluctuation in LQI values due to these random movements is obvious in Fig. 2.

\subsection{Impact of the High Power of the Base Station}

Another important aspect of any sensor network is the transmission power of its nodes. Transmission power limits the range of any given sensor. Sensor network relies upon neighbor discovery and route discovery mechanism to communicate with BS. Therefore, it is interesting to see, how different level of BS energy may affect sensor network. Scenarios 26 and 27 are different only in terms of TPL level of BS. In both the scenarios, we have 7 sensor nodes, separated by 6 meters in the straight line. Fig. 4 presents the LQI readings of each sensor with BS, in a two different networks (for ease of clarity, again only few nodes are depicted). As, we compare LQI values, we can observe, that just by increasing the TPL of the BS, the LQI between the nodes and the BS improves tremendously. Also, the lower the TPL of BS the lower is the LQI (apart from sensor which is nearest to BS). Further, we can clearly observe the difference of LQI readings of sensor which is being placed at a distance of 18 meters from the BS. Due to difference in LQI values of these sensors, sensor with BS (31 points, scenario 


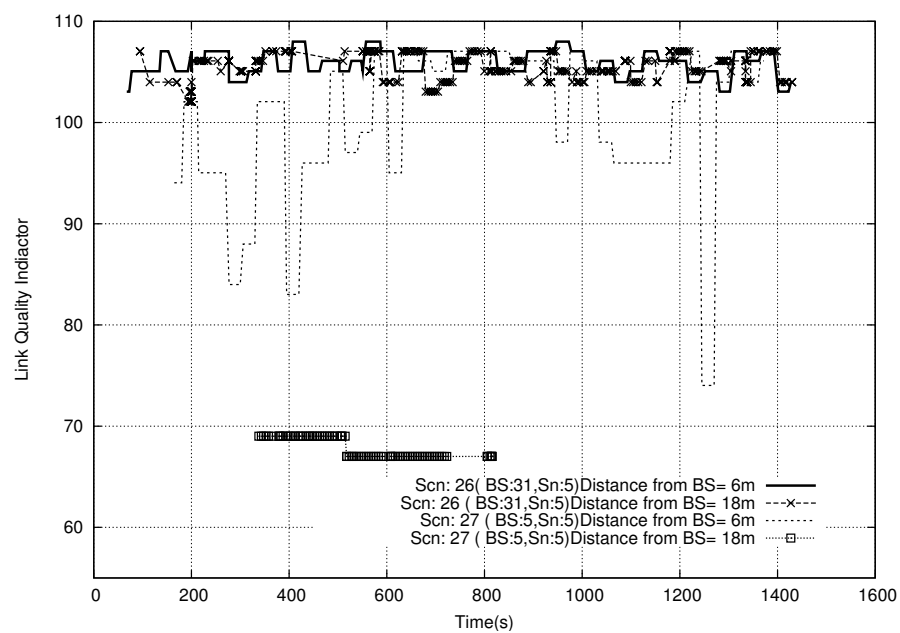

Fig. 4. Impact of high power of Base Station

26) remains connected continuously with BS, the other sensor in Scenario 27 is rather connected via its neighbours.

Higher power level for a given node leads to a natural single hop cluster, since each node sees the BS as being close (even if it is far) and consumes lot of energy because of its high transmission power level. And then, each node tries to communicate directly with the BS instead of communicating to BS via a set of hops. This raises some more issues for example in terms of traffic where a traffic can be captured by a single high power node. In fact, in the next subsection, we will magnify this effect in the grid topology and the ramification of this phenomenon.

\subsection{Impact of Different Power Level i.e Heterogeneous Sensor Network}

Table 2 presents another set of tests (Scenarios 30-40). These tests are executed in an indoor room but in an area cut-off from the public. We have used two different grids of size $4 \times 4$ and $3 \times 6$. In both cases, sensors are separated by 3 meters. In these scenarios, two TPL sets are defined as $\operatorname{SetMax}\{31\}$ and $\operatorname{SetLow}\{10,5,3\}$.

\section{Link Quality with Distance}

Here, the link quality variations are not completely due to the change in the physical properties of the channel because of the closed environment (a classroom) without any presence of people. Generally (Fig. 5), all the collected values for every combination of distance and transmission power vary between 103 to 108. Furthermore, if we refer to other kind of experiments [5, 6], 11] these values remain interesting because the packet received rate for such LQI values is high. 
Table 2. Grid Scenario Description

\begin{tabular}{|c|c|c|c|c|}
\hline Scenario & Nodes count & BS-TPL & Node-TPL & Distance \\
\hline 30 & $4 \times 4$ & 31 & 10 & 3 \\
\hline 31 & $4 \times 4$ & 10 & 10 & 3 \\
\hline 32 & $4 \times 4$ & 3 & 10 & 3 \\
\hline 33 & $4 \times 4$ & 31 & 5 & 3 \\
\hline 34 & $4 \times 4$ & 5 & 5 & 3 \\
\hline 35 & $3 \times 6$ & 31 & 10 & 3 \\
\hline 36 & $3 \times 6$ & 10 & 10 & 3 \\
\hline 37 & $3 \times 6$ & 3 & 10 & 3 \\
\hline 38 & $3 \times 6$ & 31 & 5 & 3 \\
\hline 39 & $3 \times 6$ & 5 & 5 & 3 \\
\hline 40 & $3 \times 6$ & 3 & 5 & 3 \\
\hline
\end{tabular}

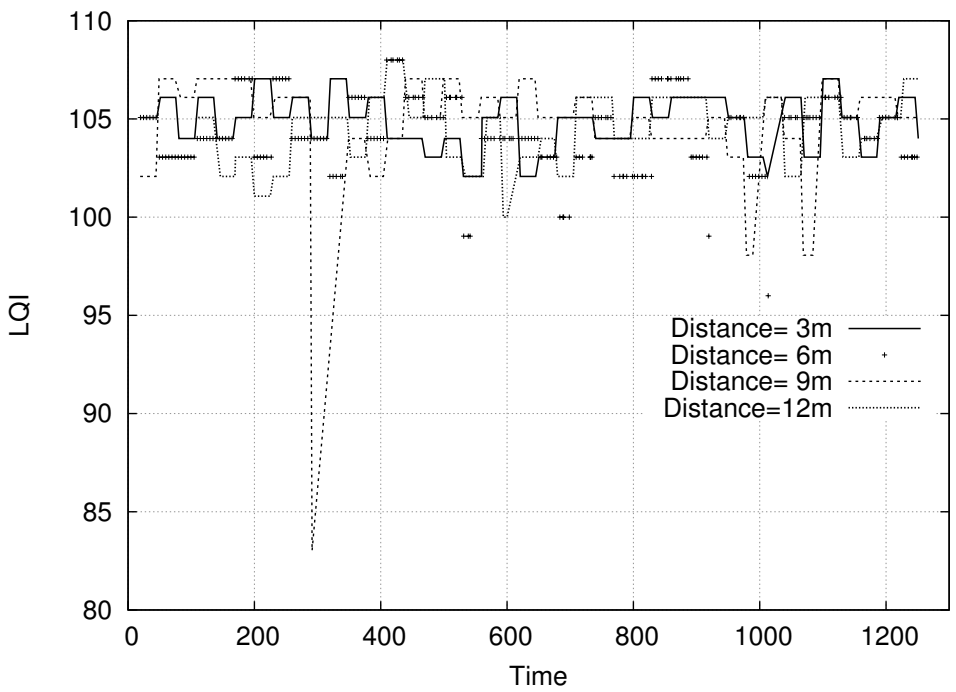

Fig. 5. LQI variation with time, scenario 33

For a given transmission power level, the LQI values are slightly different (i.e they decrease when distance from the Base Station increases); and for a given distance, these values decrease slightly when we reduce the transmission power. When the distance from BS is higher than 3 or 6 meters, we notice some dramatical decreases in the LQI variations. We also observe that the variations of LQI are more frequent with the nodes placed along the wall, than when they are placed in diagonally.

\section{Influence of BS Transmission Power on Topology}

To conduct our experiments, we have used multiHop LQI routing algorithm 12 in TinyOS, because the code for the Tmote Sky platform was available. 


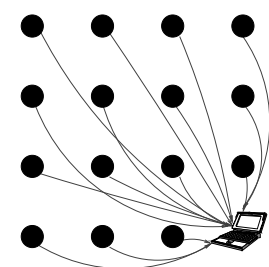

(a)

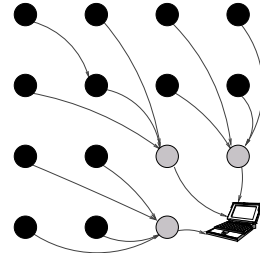

(b)

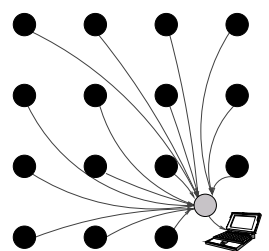

(c)

Fig. 6. BS transmission power effects

According to this algorithm, we noticed that the transmission power of the Base Station is a crucial parameter. Moreover, the BS has an important role in the network topology and the route changing. Indeed, in order to allow the nodes to choose their routes to reach the Base Station, the Base Station required to send beacon packets regularly.

As shown in Fig. 4.4. we analyze the results of these tests according to three distinct cases. The first case, when the Base Station transmits with a higher power than the power of the nodes. In this case, all the nodes note that the link quality with the BS is sufficiently high to choose direct connections (Fig. 6(a) . The second case, when the Base Station transmits with the same power than the nodes, we observed some multi-hop routes especially for the furthest nodes. The third case when the Base Station transmits with a lower power than the power of the nodes, several multi-hop connections appear with an important traffic overload on the nodes closer to the BS. (Fig. 6(b). Indeed, the routing algorithm issues that getting through these nodes constitutes the most optimal way (number of hops) and the most effective (link quality). We proved that by adding another node with a high transmission power beside the Base Station and all the traffics are transmitted via this node (Fig. 6(c)).

\section{Influence of Nodes Transmission Power on LQI and Multi-hopping}

While the routing algorithm is mainly based on the link quality, thus, varying the nodes TPL implies certainly changes in the network topology. Here, we consider the scenarios 30 to 40 to analyze these changes according to the nodes- and BS-TPL.

Fig. 7 plots the average number of hops as a function of the BS- and nodeTPL, observed in a grid of 3x6 nodes. We can note that the number of hops increases with the reduction in BS-TPL. This result endorses the observations of the preceding paragraph on BS-TPL impact. The number of hops remains reasonable (3) even with the lowest BS-TPL because the area is relatively small $(6 \times 15 m)$.

On the other hand, Fig. 8 illustrates the difference between two sets of scenarios Set $\{35,36,37\}$ and set $\{38,39,40\}$. In these two sets we used two distinct Node-TPL (respectively 10 and 5). In this figure we can clearly note that the number of multi-hop paths is always higher when the node-TPL is lower. 


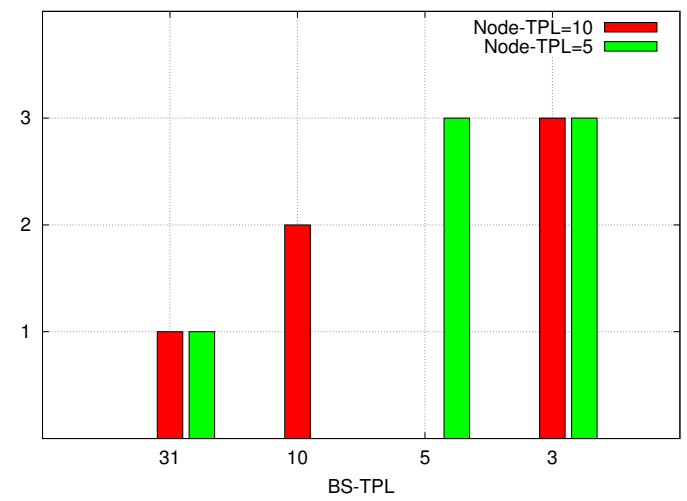

Fig. 7. Average number of hops

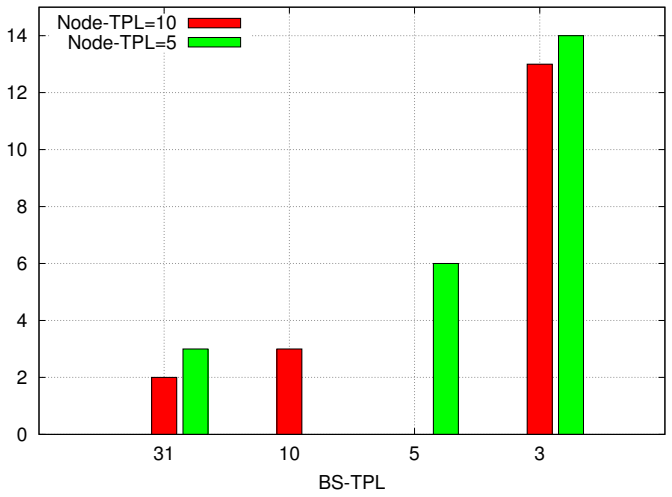

Fig. 8. Number of multi-hop routes

\section{Conclusion}

Focusing on using a commercial hardware platform in sensor systems, we carried out in this work, an experimental study on the link quality in wireless sensor networks. In the first set of experiments, we studied the LQI evolution over time and observed the dynamics of transmission channel. Very briefly, we discussed the significance of positioning of the Base Station in any given sensor network. We saw, how network is sensitive to small node displacements. With these experiences, we presented LQI time-varying and some random disturbances due to external phenomena and physical changes. It is very important to study these issues, as sensors may not be subjected to steady state deployment. Finally, we studied the impact of transmission power of BS and observed, how sensors in networks with high TPL of BS can miss-construct network topology and the effects on the connectivity between nodes and BS. We saw, how a high power node creates a natural single hop cluster. We used these observations and experiences to conduct further experiments. 
In the second set of experiments, we have also investigated the impact of nodes transmission power on the LQI which affects consequently the network topology. Indeed, with high BS-TPL and Node-TPL, often we observed only one cluster (BS as a cluster-head). When we varied the TPL between nodes (heterogeneous nodes), several clusters appeared (cluster-head with high TPL). So, it may be a possible solution to organize the network on clusters. However, such heterogeneity may affect the lifespan of these nodes and the network connectivity.

We also proved in this study that the Base Station TPL may have a misleading effect for the furthest nodes. Indeed, these nodes notice that the link quality with the BS is sufficiently high to choose direct connections. But the distance is large and the risk of packet loss might increase. Indeed, the link quality on the another direction (node to BS) is not necessarily the same because of the distance or the weak Node-TPL. As a concluding remark, routing protocols should not be entirely based on LQI.

Considering our measurement results, it seems that it may be interesting to reduce the transmission power in order to save energy, or to deploy heterogeneous nodes for topology issues. As future work, we plan to endorse these conclusions by an evaluation of the network performance such as energy-efficiency, fairness, transmission delay, etc.

\section{Acknowledgments}

The authors gratefully acknowledge material and technical support from the CNRS-SAMOVAR (Evry), IRIT-ENSEEIHT (Toulouse), National Polytechnic Institute of Toulouse (INPT) and TELECOM-SudParis. This work is also supported by the "CAPTEURS" grant, a National Telecommunication Research Network (RNRT) project.

\section{References}

1. CC2420 Radio, http://www.chipcon.com

2. Tmote Sky datasheet, http://www.moteiv.com/products/docs/tmote-sky-datasheet.pdf

3. Gungor, V.C., an Sastry, C., Song, Z., Integlia, R.: Resource-aware and link quality based routing metric for wireless sensor and actor networks. In: IEEE International Conference on Communications, 2007. ICC 2007, pp. 3364-3369 (June 2007)

4. Blumenthal, J., Grossmann, R., Golatowski, F., Timmermann, D.: Weighted centroid localization in zigbee-based sensor networks. In: IEEE International Symposium on Intelligent Signal Processing, 2007. WISP 2007, pp. 1-6 (October 2007)

5. Wahba, S.K., LaForce, K.D., Fisher, J.L., Hallstrom, J.O.: An empirical evaluation of embedded link quality. In: International Conference on Sensor Technologies and Applications, 2007. SensorComm 2007, pp. 430-435 (October 2007)

6. Lal, D., Manjeshwar, A., Herrmann, F., Uysal-Biyikoglu, E., Keshavarzian, A.: Measurement and characterization of link quality metrics in energy constrained wireless sensor networks. In: IEEE Global Telecommunications Conference, 2003. GLOBECOM 2003, vol. 1, pp. 446-452 (December 2003) 
7. Son, D., Krishnamachari, B., Heidemann, J.: Experimental analysis of concurrent packet transmissions in wireless sensor networks. In: Proceedings of the Fourth ACM SenSys Conference, Boulder, Colorado, USA, pp. 237-249. ACM, New York (2006)

8. Srinivasan, K., Dutta, P., Tavakoli, A., Levis, P.: Understanding the causes of packet delivery success and failure in dense wireless sensor networks. In: ACM SenSys. (2006)

9. Zhao, J., Govindan, R.: Understanding packet delivery performance in dense wireless sensor networks. In: SenSys 2003: Proceedings of the 1st international conference on Embedded networked sensor systems, pp. 1-13. ACM, New York (2003)

10. Polastre, J., Szewczyk, R., Culler, D.: Telos: enabling ultra-low power wireless research. In: IPSN: Information Processing in Sensor Networks, pp. 364-369 (2005)

11. Holland, M., Aures, R., Heinzelman, W.: Experimental investigation of radio performance in wireless sensor networks. In: 2nd IEEE Workshop on Wireless Mesh Networks, 2006, pp. 140-150 (2006)

12. TinyOs MultiHopLQI routing algorithm, http://www.tinyos.net/tinyos-1.x/tos/lib/MultiHopLQI 